\title{
EVALUACIÓN Y DISEÑO DE UN SISTEMA DE TRATAMIENTO DE AGUA RESIDUAL PARA LA FÁBRICA TEXTIL HILARIO
}

\section{Evaluation and Design of a Treatment System for Residual Water in the Hilario Textile Factory}

\author{
Karina Ramos, Mabel Parada*, Sofía Godoy
}

Facultad de Ciencias/Escuela de Ingeniería Química, Escuela Superior Politécnica de Chimborazo (ESPOCH), Riobamba (Ecuador), *mparada@espoch.edu.ec

\section{$\mathbf{R}$}

En el Ecuador, el Ministerio del Ambiente es el encargado de regular las descargas de efluentes líquidos residuales producidos por actividades humanas o industriales, en el caso de las industrias textiles tienen un alto impacto ambiental, debido al elevado consumo de agua y naturaleza de los diferentes contaminantes, haciendo énfasis en los colorantes. La fábrica de textiles Hilario ubicada en el cantón Guano, refleja esta problemática por lo cual es indispensable instalar un sistema de tratamiento de aguas residuales para evitar la contaminación del suelo y la consecuente disminución de su fertilidad en el área donde se descarga el efluente, mismo que erradamente es utilizado como agua para riego de la vegetación circundante a la planta de producción. Se propone un diseño de PTAR, el cual inicia con la medición del caudal y un muestreo compuesto del agua residual proveniente de los dos tipos de tela que se procesan: poliéster y poli algodón, se conoce la calidad del agua mediante una caracterización físico química y a partir de estos resultados se determina que únicamente el agua del proceso de poli algodón, presenta valores elevados en parámetros como: Sólidos Disueltos Totales (SDT), Color, DBO, DQO, Tensoactivos y Metales Pesados. Las pruebas de tratabilidad nos orientan a la selección del sistema de tratamiento más adecuado, que constará de tratamientos físico-químicos como: coagulación-floculación, sedimentación, filtración y cloración.

Palabras claves: Material compuesto, fibra de sisal, propiedades mecánicas, reforzamiento estructural.

\section{A bstract}

In Ecuador, the Ministry of the Environment is in charge of regulating the discharges of residual liquid effluents produced by human or industrial activities, in the case of textile industries they have a high environmental impact, due to the high water consumption and nature of the different pollutants, emphasizing dyes. The textile factory Hilario located in the Guano canton, reflects this problem, which is why it is essential to install a wastewater treatment system to avoid soil contamination and the consequent decrease of its fertility in the area where the effluent is discharged. Which is mistakenly used as water to irrigate the vegetation surrounding the production plant. A PTAR design is proposed, which starts with the flow measurement and a composite sample of the wastewater coming from the two types of fabric that are processed: polyester and poly cotton, the water quality is known by a physical chemical characterization and From these results it is determined that only the water of the poly cotton process, presents high values in parameters such as: Total Dissolved Solids (TDS), Color, BOD, COD, Surfactants and Heavy Metals. The treatability tests guide us to the selection of the most appropriate treatment system, which will consist of physical-chemical treatments such as: coagulation-flocculation, sedimentation, filtration and chlorination.

Keywords: Residual water, design, environmental regulation, treatment system.

Fecha de recepción: 03-nov-2017

Fecha de aceptación: 21-may-2018 


\section{INTRODUCCIÓN}

En el Ecuador, en la Sierra Central del país se encuentra el cantón Guano, llamada la "Capital Artesanal del Ecuador", en este lugar existen aproximadamente 100 talleres que fabrican todo tipo de textiles como: alfombras, chompas de cuero, sacos de lana, etc.. [1], considerando que en este lugar se desarrolla ampliamente la industria textil se ha tomado como objeto de estudio una de sus fábricas textiles para diseñar un sistema de tratamiento de aguas residuales. Las Fábricas Textiles del cantón Guano se dedica a la producción de telas: poliéster y poli algodón. El proceso de producción requiere la utilización de una gran cantidad de agua, así como una variedad de agentes químicos. El diseño de un sistema de tratamiento permitirá reducir los índices de contaminación del agua residual, con la finalidad de ajustar los parámetros de calidad del agua a lo establecido en el Texto Unificado de Legislación Secundaria del Ministerio del Ambiente (TULSMA), Libro VI, Anexo I, Recurso Agua, Tabla 3: Criterios de Calidad de Aguas para Riego Agrícola, y así estas aguas puedan ser reutilizadas para fines agrícolas.

\section{MATERIAL Y MÉTODO}

\section{Descripción del proceso productivo}

El proceso de producción textil de una fábrica textil, para la transformación de hilo crudo en tela acabada consta de tres etapas: Hilatura, Tejeduría y Acabados. Los efluentes industriales se generan en ésta última ya que constan de procesos que se realizan en húmedo. En la figura 1 , se indica la información del proceso de producción.

\section{Medición del caudal}

Previamente identificado el lugar donde convergen las aguas residuales, se proce-

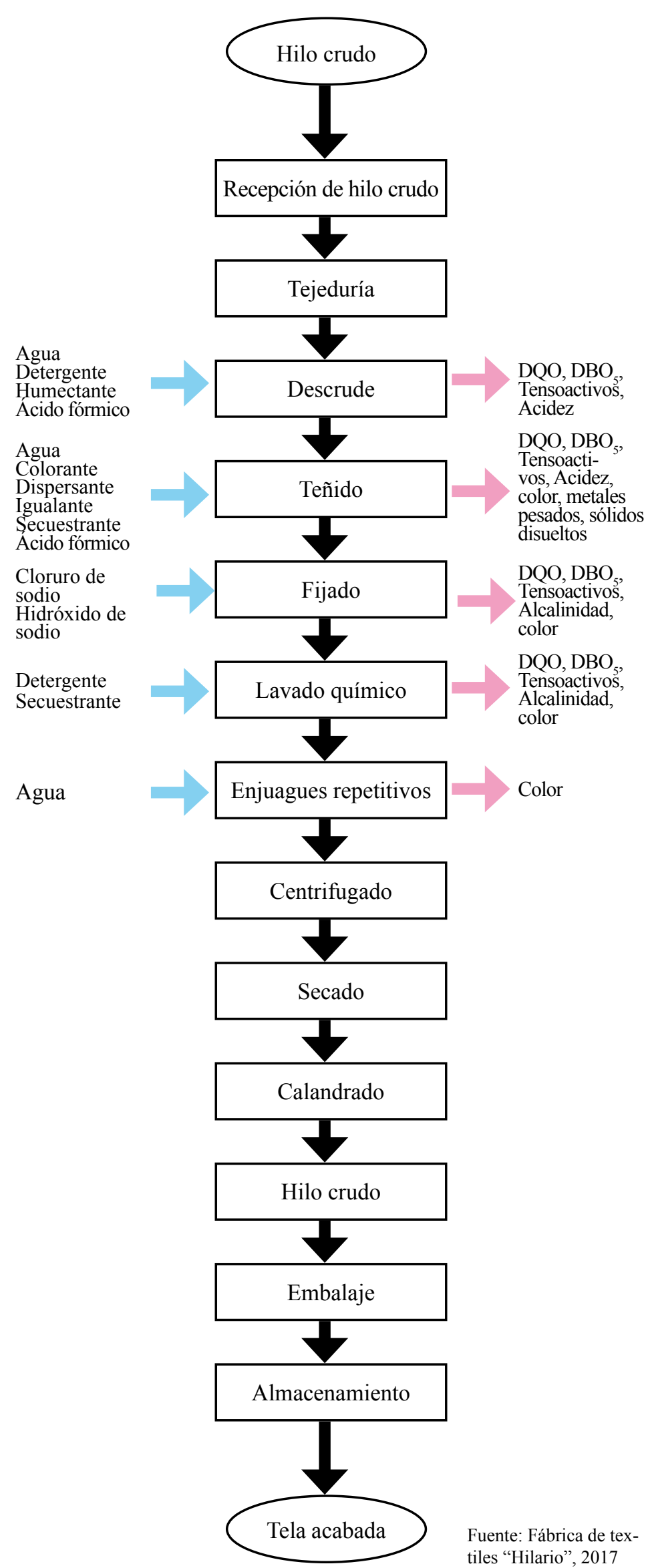

tiles "Hilario", 2017
Figura 1. Diagrama de flujo de entradas y salidas del proceso de producción de telas de la Fábrica Textil Hilario. 
dió a la medición del caudal mediante el método volumétrico basado en el manual de Métodos Normalizados para el Análisis de Agua Potable y Residuales, para determinar el caudal promedio total como indica la tabla 1.

\begin{tabular}{|c|c|}
\hline Etapas del proceso & Caudal promedio (L/s) \\
\hline Descrude & 2,77 \\
\hline Teñido & 2,76 \\
\hline Fijado & 2,78 \\
\hline Lavado químico & 2,79 \\
\hline Enjuegue 1 & 2,76 \\
\hline Enjuague 2 & 2,76 \\
\hline Caudal promedio total & $\mathbf{2 , 7 7}$ \\
\hline
\end{tabular}

Tabla 1. Caudal promedio total

El Caudal promedio total es de $2.77(\mathrm{~L} / \mathrm{s})$, el tiempo en el que se produce cada descarga es de 15 minutos, siendo 6 descargas, se tiene como resultado un volumen equivalente a 14958 litros de agua residual al día.

\section{Muestreo}

Se realizó el muestreo de tipo compuesto y de manera manual según la norma técnica ecuatoriana NTE INEN 2169:2013, siguiendo la respectiva planificación para

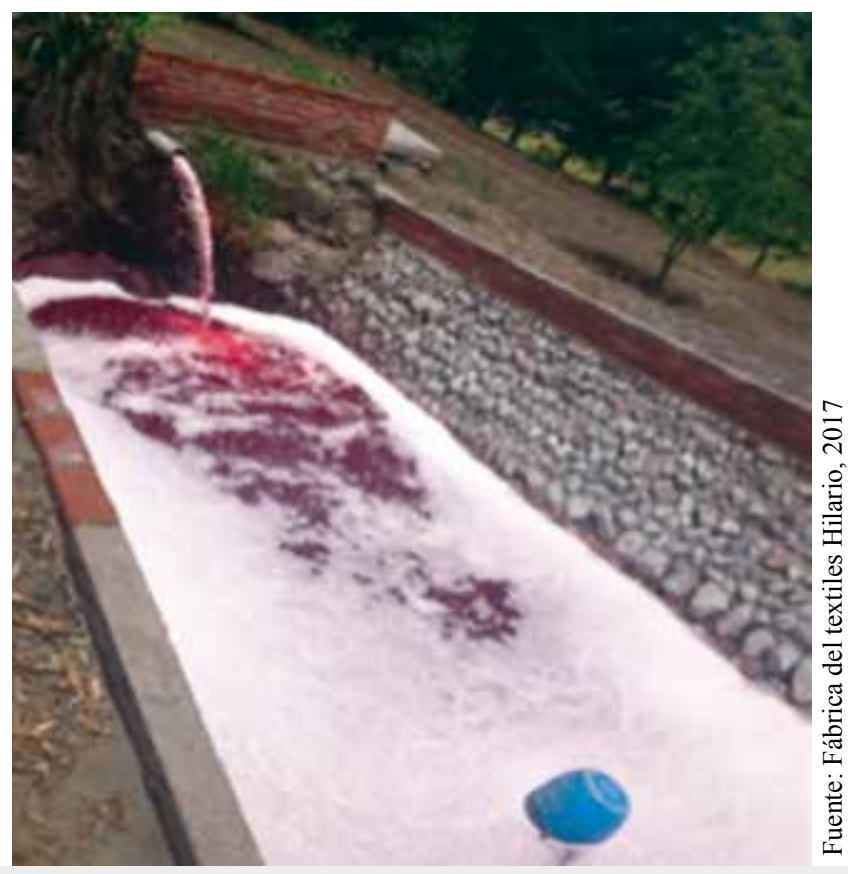

Figura 2. Tanque de almacenamiento de aguas residuales de la Fábrica Textil Hilario. obtener 2 litros de muestra compuesta al día como se observa en la figura 2 . El muestreo se lo realizó en los días que se ejecutan los procesos húmedos, recolectando 0.333 litros de agua residual de cada una de las 6 descargas del proceso productivo, para que la muestra de 2 litros sea representativa en cuanto a la composición de los contaminantes presentes en la misma.

\section{Caracterización físico-química del agua residual}

Se realizó la caracterización de las muestras del agua residual proveniente de los dos procesamientos tanto para tela poliéster como poli algodón, determinándose la media aritmética de cada procesamiento para mostrarse en la columna de resultados, basándose en el manual de Métodos Normalizados para el Análisis de Agua Potable y Residuales y en el método $\mathrm{HACH}$, como se muestra en las Tablas 2 y 3.

Como se indica en la Tabla 2, todos los valores cumplen con la normativa, algunos de ellos están muy por debajo del límite establecido, incluso parámetros como color y tensoactivos que son los principales contaminantes de este tipo de efluentes.

En la tabla 3, se puede observar que solamente el parámetro de sólidos sedimentables cumple con la normativa, los demás valores están por encima del límite establecido.

\section{Pruebas de Tratabilidad}

a) Prueba de jarras: se realizó previamente pruebas con coagulantes metálicos y polielectrolitos para verificar o descartar su utilidad para este tipo de agua residual, determinándose que solamente el sulfato de aluminio reaccionó favorablemente, de ahí la necesidad de encontrar la dosificación precisa de éste coagulante para lo cual se efectuaron prueba de jarras, resultando una dosis óptima de 10 


\begin{tabular}{|l|c|c|c|c|c|}
\hline \multicolumn{1}{|c|}{ Parámetro } & Unidad & Método & Resultado (x) & Límite & Cumple \\
\hline Temperatura & ${ }^{\circ} \mathrm{C}$ & $2500-\mathrm{A}$ & 19.6 & --- & - -- \\
\hline Conductividad & $\mathrm{mmhos} / \mathrm{cm}$ & $2510-\mathrm{B}$ & 19.6 & 0.7 & Sí \\
\hline Color & $\begin{array}{c}\text { Unidades de } \\
\text { color }\end{array}$ & $2120-\mathrm{C}$ & $\begin{array}{c}\text { Inapreciable en } \\
\text { dilución: } 1 / 20\end{array}$ & $\begin{array}{c}\text { Inapreciable en } \\
\text { dilución: } 1 / 20\end{array}$ & Sí \\
\hline Turbuedad & $\mathrm{UNT}$ & $2130-\mathrm{B}$ & 67 & --- & --- \\
\hline Sólidos totales & $\mathrm{mg} / \mathrm{L}$ & $2540-\mathrm{B}$ & 566 & 1600 & Sí \\
\hline Sólidos suspendidos totales & $\mathrm{mg} / \mathrm{L}$ & $2540-\mathrm{D}$ & 88.3 & 130 & Sí \\
\hline Sólidos disueltos totales & $\mathrm{mg} / \mathrm{L}$ & $2510-\mathrm{C}$ & 257.7 & 450 & Sí \\
\hline Sólidos sedimentables & $\mathrm{mg} / \mathrm{L}$ & $2540-\mathrm{F}$ & $<1$ & 1.0 & Sí \\
\hline Potencial de hidrógeno & $\mathrm{UND}$ & $4500-\mathrm{B}$ & 6.55 & $6-9$ & Sí \\
\hline $\begin{array}{l}\text { Demanda química de } \\
\text { oxígeno }\end{array}$ & $\mathrm{mg} / \mathrm{L}$ & $5220-\mathrm{D}$ & 161 & 200 & Sí \\
\hline $\begin{array}{l}\text { Demanda bioquñimica de } \\
\text { oxígeno } 5 \text { días }\end{array}$ & $\mathrm{mg} / \mathrm{L}$ & $5210-\mathrm{B}$ & 78 & 100 & Sí \\
\hline Tensoactivos & $\mathrm{mg} / \mathrm{L}$ & $5540-\mathrm{C}$ & 0.43 & 0.5 & Sí \\
\hline Cobre & $\mathrm{mg} / \mathrm{L}$ & $3500 \mathrm{Cu}-\mathrm{B}$ & 0.020 & 0.2 & Sí \\
\hline Niquel & $\mathrm{mg} / \mathrm{L}$ & $3500 \mathrm{Ni}, 3111-\mathrm{B}$ & 0.017 & 0.2 & Sí \\
\hline Cromo & $\mathrm{mg} / \mathrm{L}$ & $\mathrm{Cr}+68023$ & 0.065 & 0.1 & Sí \\
\hline Aluminio & $\mathrm{mg} / \mathrm{L}$ & $3500 \mathrm{Al}-\mathrm{B}$ & 0.55 & 5.0 & Sí \\
\hline Hierro & $\mathrm{mg} / \mathrm{L}$ & $3500 \mathrm{Al}-\mathrm{B}$ & 0.87 & 5.0 & Sí \\
\hline
\end{tabular}

Fuente: Laboratorio de Análisis Técnicos de la Facultad de Ciencias, CESTTA y laboratorio de servicios ambientales UNACH, 2017

Tabla 2. Caracterización físico -química del agua residual proveniente del procesamiento de tela poliéster (100 \%) y comparación con los límites de la normativa ambiental.

\begin{tabular}{|c|c|c|c|c|c|}
\hline Parámetro & Unidad & Método & Resultado (x) & Límite & Cumple \\
\hline Temperatura & ${ }^{\circ} \mathrm{C}$ & $2500-\mathrm{A}$ & 19,7 & --- & --- \\
\hline Conductividad & $\mathrm{mmhos} / \mathrm{cm}$ & $2510-B$ & 12,5 & 0,7 & No \\
\hline Color & $\begin{array}{l}\text { Unidades de } \\
\text { color }\end{array}$ & $2120-\mathrm{C}$ & $\begin{array}{l}10000 \text { Aprpeciable } \\
\text { en disolución } 1 / 20\end{array}$ & $\begin{array}{c}\text { Inapreciable en } \\
\text { dilución : } 1 / 20\end{array}$ & No \\
\hline Turbuedad & UNT & 2130-B & 1716,0 & --- & --- \\
\hline Sólidos totales & $\mathrm{mg} / \mathrm{L}$ & $2540-\mathrm{B}$ & 8026,0 & 1600 & No \\
\hline Sólidos suspendidos totales & $\mathrm{mg} / \mathrm{L}$ & 2540-D & 320 & 130 & No \\
\hline Sólidos disueltos totales & $\mathrm{mg} / \mathrm{L}$ & $2510-\mathrm{C}$ & 7675,0 & 450 & No \\
\hline Sólidos sedimentables & $\mathrm{mg} / \mathrm{L}$ & $2540-\mathrm{F}$ & $<1$ & 1,0 & No \\
\hline Potencial de hidrógeno & UND & $4500-\mathrm{B}$ & 12,68 & $6-9$ & No \\
\hline $\begin{array}{l}\text { Demanda química de } \\
\text { oxígeno }\end{array}$ & $\mathrm{mg} / \mathrm{L}$ & 5220-D & 6843,0 & 200 & No \\
\hline $\begin{array}{l}\text { Demanda bioquñimica de } \\
\text { oxígeno ( } 5 \text { días }\end{array}$ & $\mathrm{mg} / \mathrm{L}$ & 5210-B & 2648,0 & 100 & No \\
\hline Tensoactivos & $\mathrm{mg} / \mathrm{L}$ & $5540-\mathrm{C}$ & 9,93 & 0,5 & No \\
\hline Cobre & $\mathrm{mg} / \mathrm{L}$ & $3500 \mathrm{Cu}-\mathrm{B}$ & 6,94 & 0,2 & No \\
\hline Niquel & $\mathrm{mg} / \mathrm{L}$ & $3500 \mathrm{Ni}, 3111-\mathrm{B}$ & 8,64 & 0,2 & No \\
\hline Cromo & $\mathrm{mg} / \mathrm{L}$ & $\mathrm{Cr}^{+6} 8023$ & 1,57 & 0,1 & No \\
\hline Aluminio & $\mathrm{mg} / \mathrm{L}$ & 3500 Al-B & 5,67 & 5,0 & No \\
\hline Hierro & $\mathrm{mg} / \mathrm{L}$ & $3500 \mathrm{Al}-\mathrm{B}$ & 6,47 & 5,0 & No \\
\hline
\end{tabular}

Fuente: Laboratorio de Análisis Técnicos de la Facultad de Ciencias, CESTTA y laboratorio de servicios ambientales UNACH, 2017

Tabla 3. Caracterización físico -química del agua residual proveniente del procesamiento de tela poliéster (100 \%) y comparación con los límites de la normativa ambiental. 


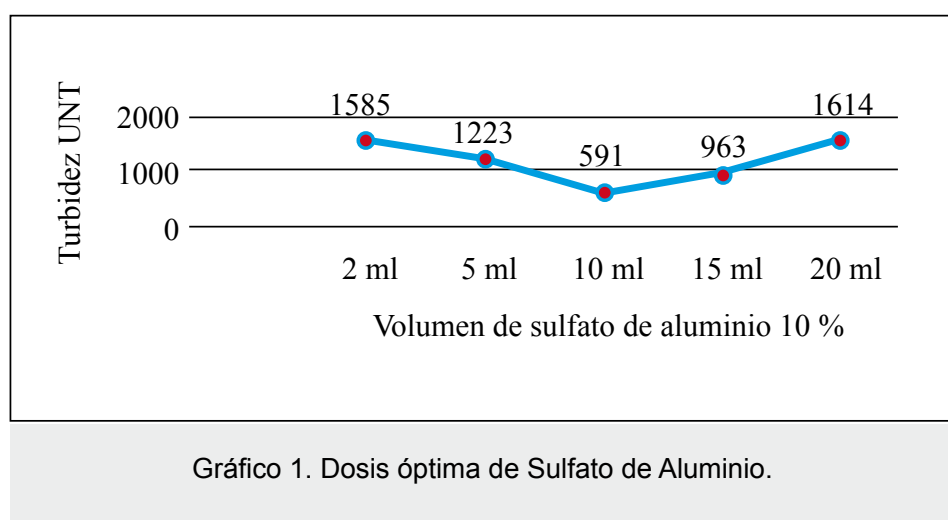

$\mathrm{ml}$ de Sulfato de Aluminio al $10 \%$ por cada litro de agua residual o 1000 ppm (como se muestra en el gráfico 1).

b) Sedimentación: el tiempo de sedimentación de la muestra estudiada fue de 2 horas, tiempo en el cual se obtuvo flóculos de mejor tamaño y textura. Así como también se obtuvo el volumen de lodos siendo $88 \mathrm{ml}$ de lodos por cada litro de agua residual. En la fase líquida se verificó la reducción de la carga contaminante mediante análisis de laboratorio. c) Filtración: el filtro se simuló con carbón activado y arena de manera que éstos absorban los contaminantes que aún no fueron removidos en procesos anteriores. d) Cloración: se determinó la dosis óptima del hipoclorito de calcio de $30 \mathrm{mg} / 1$ con un tiempo de reacción de 30 minutos.

\section{Caracterización físico-química del agua tratada.}

Para verificar que mejoró la calidad del agua tratada se realizó una caracterización final.

Como se puede observar en la tabla 4 , todos los valores de los parámetros tanto físicos como químicos del agua tratada se encuentran dentro del límite establecido por el TULSMA.

\begin{tabular}{|c|c|c|c|c|c|c|}
\hline Parámetro & Unidad & Método & Agua residual & Agua tratada & Límite & Cumple \\
\hline Temperatura & ${ }^{\circ} \mathrm{C}$ & $2500-A$ & 19,7 & 19,0 & --- & --- \\
\hline Conductividad & $\mathrm{mmhos} / \mathrm{cm}$ & 2510-B & 12,5 & 0,46 & 0,7 & Sí \\
\hline Color & $\begin{array}{l}\text { Unidades de } \\
\text { color }\end{array}$ & $2120-\mathrm{C}$ & $\begin{array}{l}10000 \text { apreciable } \\
\text { en disulución: } 1 / 20\end{array}$ & $\begin{array}{c}475 \text { inapreciable } \\
\text { en disolución } 1 / 20\end{array}$ & $\begin{array}{l}\text { Inapreciable en } \\
\text { disolución } 1 / 20\end{array}$ & Sí \\
\hline Turbiedad & UNT & 2130-B & 1761,0 & 29 & -- & --- \\
\hline Sólidos totales & $\mathrm{mg} / \mathrm{L}$ & $2540-B$ & 8026,0 & 593 & 1600 & $\overline{\text { Sí }}$ \\
\hline $\begin{array}{l}\text { Sólidos suspendidos } \\
\text { totales }\end{array}$ & $\mathrm{mg} / \mathrm{L}$ & 2540-D & 320 & 81 & 130 & Sí \\
\hline $\begin{array}{l}\text { Sólidos disueltos } \\
\text { totales }\end{array}$ & $\mathrm{mg} / \mathrm{L}$ & $2510-\mathrm{C}$ & 7675,0 & 289 & 450 & Sí \\
\hline $\begin{array}{l}\text { Sólidos } \\
\text { sedimentarios }\end{array}$ & $\mathrm{mg} / \mathrm{L}$ & $2540-\mathrm{F}$ & $<1$ & $<1$ & 1,0 & Sí \\
\hline $\begin{array}{l}\text { Potencial de } \\
\text { hidrógeno }\end{array}$ & UND & $4500-B$ & 12,68 & 7,82 & $6-9$ & Sí \\
\hline $\begin{array}{l}\text { Demanda química } \\
\text { de oxígeno }\end{array}$ & $\mathrm{mg} / \mathrm{L}$ & 5220-D & 6843,0 & 182 & 200 & Sí \\
\hline $\begin{array}{l}\text { Demanda química } \\
\text { de oxígeno }\end{array}$ & $\mathrm{mg} / \mathrm{L}$ & $5210-\mathrm{B}$ & 2648,0 & 81 & 100 & Sí \\
\hline Tensoactivos & $\mathrm{mg} / \mathrm{L}$ & 5540-C & 9,93 & 0,38 & 0,5 & Sí \\
\hline Cobre & $\mathrm{mg} / \mathrm{L}$ & $3500 \mathrm{Cu}-\mathrm{B}$ & 6,94 & 0,11 & 0,2 & Sí \\
\hline Níquel & $\mathrm{mg} / \mathrm{L}$ & $3500 \mathrm{Ni}, 3111-\mathrm{B}$ & 8,64 & 0,13 & 0,2 & Sí \\
\hline Cromo & $\mathrm{mg} / \mathrm{L}$ & $\mathrm{Cr}^{+6} 8023$ & 1,57 & 0,09 & 0,1 & Sí \\
\hline Aluminio & $\mathrm{mg} / \mathrm{L}$ & $3500 \mathrm{Al}-\mathrm{B}$ & 5,67 & 3,7 & 5,0 & Sí \\
\hline Hierro & $\mathrm{mg} / \mathrm{L}$ & $3500 \mathrm{Fe}-\mathrm{B}$ & 6,47 & 0,9 & 5,0 & Sí \\
\hline Cloro residual & $\mathrm{mg} / \mathrm{L}$ & yodométrico & & 0,92 & $0,3-1,5$ & Sí \\
\hline
\end{tabular}

Fuente: Laboratorio de Análisis Técnicos de la Facultad de Ciencias, CESTTA y laboratorio de servicios ambientales UNACH, 2017 


\section{Resultados del porcentaje de remoción obtenidos después de las pruebas de tratabilidad.}

Con los resultados del agua tratada es preciso conocer el porcentaje de remoción de cada uno de los contaminantes, para lo cual se toma en consideración los valores de la caracterización inicial del agua residual y el resultado obtenido después del tratamiento. En la tabla 5, se observa la disminución de los parámetros con sus respectivos porcentajes de remoción, mismos que tienen valores aceptables lo que permite estimar que la calidad del agua mejoró después del tratamiento y está dentro de lo que exige la normativa ambiental.

Cálculos de ingeniería para el diseño del sistema de tratamiento del agua residual de la fábrica textil Hilario.

El sistema de tratamiento del agua residual para ésta fábrica textil operará al final de la jornada de trabajo diaria, es decir una vez que se hayan producido las 6 descargas de agua residual provenientes del procesamiento de tela poli algodón que es la que por su carga contaminante se encuentra fuera de normativa ambiental. Para los cálculos de ingeniería se debe considerar el caudal de diseño que tiene un valor de 17949.6 litros al día, el cual fue determinado a partir del caudal promedio relacionado con un factor de mayorización de $20 \%$ según los euro códigos de ingeniería civil para este tipo de volúmenes de agua. El sistema de tratamiento del agua residual consta de: Un tanque de almacenamiento ya existente en la fábrica con un volumen de $20 \mathrm{~m}^{3}$.Un tanque circular de sedimentación con un volumen de $18.85 \mathrm{~m}^{3}$, valor que se calculó relacionando los diferentes parámetros de diseño como el área, radio, diámetro, volumen de la parte cilíndrica y volumen de la parte cónica del sedimentador, el tiempo de retención hidráulica es de 2 horas el mismo que se determinó experimentalmente en el cono imhoff y coincide con los valores de tiempo para la retención hidráulica de la sedimentación según bibliografía de Aurelio Hernández. Un filtro convencional lento con una tasa de filtración de $7 \mathrm{~m}^{3} / \mathrm{m}^{2}$ día, tomado de la tabla de clasificación de los filtros según la bibliografía de Jorge

\begin{tabular}{|c|c|c|c|c|}
\hline Parámetro & Unidad & Agua residual & Agua tratada & $\begin{array}{l}\text { Porcentaje de } \\
\text { remoción }(\%)\end{array}$ \\
\hline Temperatura & ${ }^{\circ} \mathrm{C}$ & 19,7 & 19,0 & --- \\
\hline Conductividad & $\mathrm{mmhos} / \mathrm{cm}$ & 12,5 & 0,46 & 96,32 \\
\hline Color & Unidades de color & $\begin{array}{l}10000 \text { apreciable en } \\
\text { disulución: } 1 / 20\end{array}$ & $\begin{array}{l}475 \text { inapreciable en } \\
\text { disolución } 1 / 20\end{array}$ & 95,25 \\
\hline Turbiedad & UNT & 1761,0 & 29 & 98,35 \\
\hline Sólidos totales & $\mathrm{mg} / \mathrm{L}$ & 8026,0 & 593 & 92,61 \\
\hline Sólidos suspendidos totales & $\mathrm{mg} / \mathrm{L}$ & 320 & 81 & 74,69 \\
\hline Sólidos disueltos totales & $\mathrm{mg} / \mathrm{L}$ & 7675,0 & 289 & 96,23 \\
\hline $\begin{array}{l}\text { Sólidos } \\
\text { sedimentarios }\end{array}$ & $\mathrm{mg} / \mathrm{L}$ & $<1$ & $<1$ & --- \\
\hline $\begin{array}{l}\text { Potencial de } \\
\text { hidrógeno }\end{array}$ & UND & 12,68 & 7,82 & 38,33 \\
\hline Demanda química de oxígeno & $\mathrm{mg} / \mathrm{L}$ & 6843,0 & 182 & 97,34 \\
\hline $\begin{array}{l}\text { Demanda bioquímica de } \\
\text { oxígeno ( } 5 \text { días) }\end{array}$ & $\mathrm{mg} / \mathrm{L}$ & 2648,0 & 81 & 96,94 \\
\hline Tensoactivos & $\mathrm{mg} / \mathrm{L}$ & 9,93 & 0,38 & 96,17 \\
\hline Cobre & $\mathrm{mg} / \mathrm{L}$ & 6,94 & 0,11 & 98,41 \\
\hline Níquel & $\mathrm{mg} / \mathrm{L}$ & 8,64 & 0,13 & 98,49 \\
\hline Cromo & $\mathrm{mg} / \mathrm{L}$ & 1,57 & 0,09 & 94,27 \\
\hline Aluminio & $\mathrm{mg} / \mathrm{L}$ & 5,67 & 3,7 & 34.74 \\
\hline Hierro & $\mathrm{mg} / \mathrm{L}$ & 6,47 & 0,9 & 86,09 \\
\hline
\end{tabular}

Fuente: Laboratorio de Análisis Técnicos de la Facultad de Ciencias, CESTTA y laboratorio de servicios ambientales UNACH, 2017 


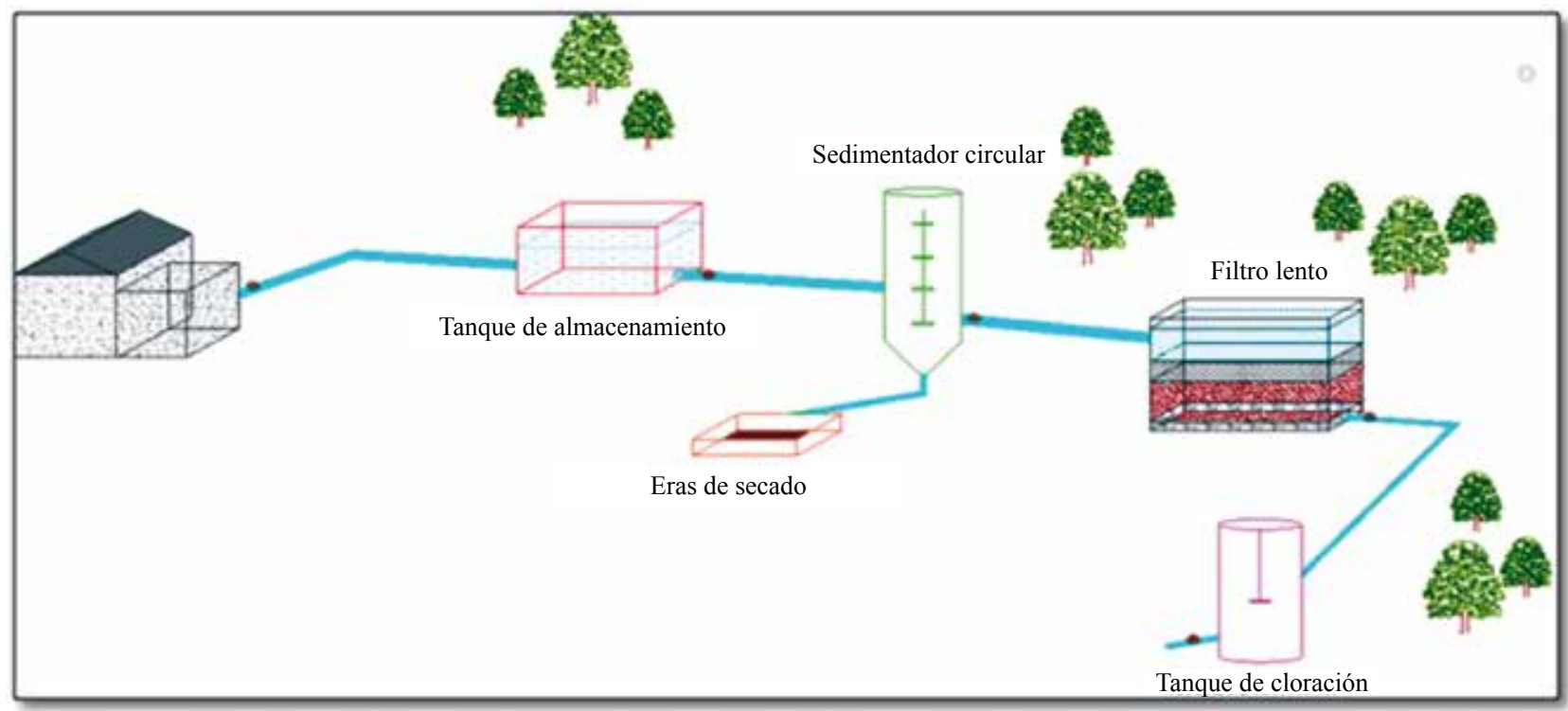

Figura 3. Vista frontal del sistema de tratamiento del agua residual para la fábrica de textiles "Hilario"

Arboleda Valencia, se calculó parámetros como el área, altura y longitud, para conocer el volumen del filtro de $26.27 \mathrm{~m}^{3}$, con un tiempo de retención de 12 horas y un sistema de drenaje y soporte con las siguientes especificaciones: espacio entre laterales: $1.20 \mathrm{~m}$, espacio entre orificios del tubo: $0.15 \mathrm{~m}$, diámetro del orificio: $3 \mathrm{~mm}$. Finalmente un tanque de cloración con un volumen de $17.97 \mathrm{~m}^{3}$, la potencia requerida para la operación de mezclado es de $1.6 \mathrm{Hp}$ con un tiempo de 20 segundos, considerando el gradiente de velocidad de $250 \mathrm{~s}^{\wedge}(-1)$ para el proceso de mezclado según la bibliografía de Metcalf-Eddy.

\section{Propuesta del diseño del sistema de tratamiento del agua residual para la fábrica textil Hilario.}

El presente estudio proviene de una necesidad real de la fábrica de textiles Hilario, debido a la degradación de la calidad del suelo que provoca la descarga del agua residual en el entorno, sin previo tratamiento. Es importante señalar que previamente se realizó el levantamiento topográfico del terreno, determinándose que el transporte del agua de una unidad a otra será por gravedad.

El proceso comienza cuando el agua almacenada pasa a un tanque sedimentador circular donde se lleva a cabo el proceso de coagulación floculación, con la ayuda de un mezclador mecánico se homogeniza el medio con el coagulante. A continuación mediante la sedimentación los flóculos adheridos con partículas contaminantes se depositan en el fondo del tanque sedimentador al cabo de 2 horas, para posteriormente ser extraídos para su respectivo tratamiento. Posteriormente el agua pasa a un filtro convencional lento donde gracias al lecho filtrante constituido por arena y sobre todo carbón activado granular se podrá remover el color y metales pesados que no fueron removidos en procesos anteriores .El agua pasa a un tanque circular de cloración, ya que el color aun es persistente, por eso la utilización de hipoclorito de calcio. Finalmente se descarga el agua tratada a los terrenos aledaños a la fábrica, con los parámetros ajustados a lo que exige la normativa ambiental.

\section{RESULTADOS}

De acuerdo a la caracterización físico-química del agua residual proveniente de los dos tipos de tela que se producen comúnmente en una fábrica textil, se identificó que el agua del procesamiento de tela poliéster $(100 \%)$ no necesitan tratarse ya que los parámetros determinados están dentro de los límites permisibles del TULSMA: Tabla 3. Criterios de calidad de aguas para riego agrícola y Tabla 9. Límites de descarga a un cuerpo de agua dulce.

Al contrario el agua residual que procede del procesamiento de tela poli 
algodón (65/35 \%) necesita tratarse ya que los parámetros como: Conductividad (12.5 milimhos/cm), Color (10000 Units PtCo), Turbiedad (1761 UNT),ST(8026 mg/l), SST(320 mg/l), SDT(7675 mg/l),pH(12.68),DQO(6 $843 \mathrm{mg} / \mathrm{l})$, DBO_5(2648 mg/l), Tensoactivos $\quad(9.93 \mathrm{mg} / \mathrm{l})$, Cobre $\quad(6.94$ $\mathrm{mg} / \mathrm{l})$, Níquel(8.64 mg/l), Cromo (1.57 $\mathrm{mg} / \mathrm{l}$ ), Aluminio ( $5.67 \mathrm{mg} / \mathrm{l}$ ), Hierro $(6.47 \mathrm{mg} / \mathrm{l})$, se encuentran fuera de los límites permisibles del TULSMA.

Con fundamento en estos datos se optó por realizar pruebas de tratabilidad al agua residual del proceso de poli algodón de un color fuerte, considerando que éste presenta valores más altos en relación a los colores bajos y medios. Se utilizaron coagulantes metálicos como el sulfato de aluminio, en diferentes concentraciones y volúmenes y tuvo una reacción favorable en la calidad del agua residual, reduciendo parámetros como: color, $\mathrm{pH}$, turbiedad, conductividad.

También se realizaron pruebas de efectividad utilizando polielectrolitos aniónicos y catiónicos en diferentes concentraciones y volúmenes, con la finalidad de comprobar y medir la eficiencia de su utilización, pero ninguno presentó una reacción favorable para el agua residual objeto de estudio, por lo que se descartó su uso.

Para encontrar la dosis óptima de sulfato de aluminio (gráfico 1), se procedió a simular el proceso de coagulaciónfloculación mediante la prueba de jarras, se puede observar que el sulfato de aluminio a una concentración de 500 ppm consigue mejorar la calidad del agua: el color, $\mathrm{pH}$, turbidez y conductividad, tienden a disminuir de manera significativa. Se descarta la utilidad del Cloruro de poli aluminio (PAC), Cloruro férrico, Sulfato férrico y el Sulfato ferroso para éste tipo de agua residual ya que no tienen ningún efecto sobre la misma, debido a que algunos de ellos tienen rangos de $\mathrm{pH}$ entre 4 y 7 para alcanzar una coagulación óptima, y debido a la alcalinidad que presenta el agua estudiada se necesitaría de grandes volúmenes de ácidos fuertes para ajustar el rango de $\mathrm{pH}$.

Se simularon procesos de sedimentación y procesos de filtración y cloración para conocer qué tan efectivos son éstos para disminuir la carga contaminante en este tipo de agua residual, obteniéndose resultados favorables como la remoción de color, DQO, turbiedad.

De acuerdo a las pruebas de tratabilidad, el sistema de tratamiento del agua residual está constituido por un proceso de coagulación floculación con la dosis óptima de sulfato de aluminio y un tiempo de mezclado de $150 \mathrm{rpm}$ durante 5 minutos para homogenizar el medio con el coagulante es decir una agitacion rápida y una agitación lenta de $40 \mathrm{rpm}$ durante $15 \mathrm{~min}$ para favorecer la formación de flóculos, determinado de manera experimental a nivel de laboratorio mediante un agitador mecánico;posteriormente la sedimentación tendrá un tiempo de retención de 2 horas para que sedimenten los flóculos seguido de una filtración en un lecho de carbón activado y arena aprovechando la capacidad absorbente de éstos materiales. Finalmente, el agua pasará a un proceso de cloración con la dosis óptima de hipoclorito de calcio con el fin de remover el color del agua residual que no pudo ser eliminado en los procesos anteriores.

\section{DISCUSIÓN}

La caracterización físico-química del agua residual reveló que solamente el agua proveniente del procesamiento de tela poli algodón $(65 / 35 \%)$ necesita tratarse ya que los parámetros se encuentran fuera de los límites permisibles por el TULSMA, como se indica en la tabla 3. En la actualidad existen diferentes alternativas de solución para el tratamiento de efluentes textiles como: Oxidación con reactivo de Fenton, Tratamiento con ozono, Degradación fotoquímica, Degradación electroquímica, entre otros. Si bien estos procesos son eficaces en cuanto a la remoción de colorantes, su principal limitante es el elevado costo de su implementación así como la formación de subproductos, por lo que el presente diseño opta por procesos conocidos, asequibles, compatibles con el medio ambiente y accesibles económicamente.

Posterior a la caracterización se aplica las pruebas de tratabilidad en el agua residual, las cuales tienen por finalidad determinar si los contaminantes presentes en un efluente son susceptibles a ser eliminados mediante el sistema de tratamiento de agua seleccionado, además 
permiten la determinación de dosis precisas para garantizar el mínimo gasto de insumos así como los parámetros de diseño, el tamaño y el tipo de las diferentes unidades que van a conforman la planta de tratamiento de agua ,se efectuaron pruebas de tratabilidad como: prueba de jarras, sedimentación, filtración y cloración. Posteriormente se caracterizó el agua tratada y los resultados se los comparó con la normativa ambiental comprobando así, que la calidad del agua mejoró de manera que se ajusta a lo exigido por la normativa ambiental, datos que se pueden observar en la tabla 4 , además se determinó el porcentaje de remoción de cada uno de los contaminantes como se indica en la tabla 5, y finalmente se puede apreciar en la figura 3 el sistema de tratamiento con cada una de las unidades que lo conforman.

\section{CONCLUSIONES}

La caracterización del agua residual proveniente del procesamiento del tipo de tela poliéster $(100 \%)$ revela que todos los valores cumplen con la normativa, la razón de éstos resultados está ligada directamente a las propiedades químicas de la tela como: la elevada sensibilidad a los álcalis fuertes, excelente resistencia a los agentes oxidantes y la utilización de colorantes dispersos que tienen un alto grado de fijación, propiedades que fueron comprobados a nivel de laboratorio .Al contrario el efluente del procesamiento de tela poli algodón $(65 / 35 \%)$ necesita tratarse ya que los demás valores están por encima del límite establecido por la normativa ambiental.

Las pruebas de tratabilidad seleccionadas como: prueba de jarras, sedimentación, filtración lenta y cloración permitieron determinar la dosis óptima de los diferentes compuestos químicos necesarios para conseguir la reducción de los parámetros de calidad del agua. Fundamentándose en estos datos y con la identificación de variables del proceso como el caudal de diseño de $2.24 \mathrm{~m} 3 / \mathrm{h}$, se diseñó un sistema de tratamiento de aguas residuales con el que se obtuvo una remoción de color del $95.25 \%$, una reducción de turbiedad de $98.35 \%$, una remoción de ST de $92.61 \%$, una remoción de SDT de $96.23 \%$, una remoción de DQO de 97.34\%, una remoción de DBO5 de $96.94 \%$, una remoción de cobre de $98.41 \%$ y una remoción de níquel de $98.49 \%$.Con lo cual se comprueba que el diseño de tratamiento planteado permite cumplir con lo establecido en el Texto Unificado de Legislación Secundaria del Ministerio del Ambiente (TULSMA) y así reutilizar el agua tratada con fines agrícolas atesorando el medio ambiente y mitigando el impacto ambiental que generan este tipo de agua residuales industriales.

\section{AGRADECIMIENTO}

El presente trabajo de investigación fue realizado bajo la supervisión de la Ing. Mabel Parada y el Ing. Marco Chuiza, a quienes expreso mi más profundo agradecimiento por hacer posible la realización de este estudio, así como a la Fábrica de textiles Hilario por su colaboración en las tareas de campo, y la aportación de toda la información necesaria.

1. Daros G, La contaminación de aguas en Ecuador: Industria Textil, 2a Ed. Quito: Lavigne, 2005, p.5.

2. Asociación Industrial Textil del Proceso Algodonero (AITPA), "El sector textil de proceso algodonero presenta sus resultados a la asamblea general". [Online] Disponible en: http://www.aitpa.es/industria/ text-mundo.htm. Ultimo acceso: 19 Nov, 2002.

3. Ecuador. TEXTO UNIFICADO DE LEGISLACIÓN SECUNDARIA DEL MINISTERIO DEL AMBIENTE (TULSMA).Norma de calidad ambiental y de descarga de efluentes, recurso agua. Quito-Ecuador. Libro VI, Anexo I. 2016. Pp.14-24.

4. Environmental Protection Agency (EPA), Waste Water Collection, Treatment and Storage. USA: EPA, 1999. 
5. European Commission, Institute for Prospective Technological Studies, Integrated Pollution Prevention and Control (IPPC), Reference Document on Best Available Techniques for the textiles industry, Sevilla, 2002.

6. H. Van der Roest, A Van Bentem, and D. Lawrence, "BRM-technology in municipal wastewater treatment: challenging the traditional treatment technologies", Water Science \& technology, Vol. 45, pp. 273 - 280, 2002.

7. Lockuán F, La industria textil: Control de calidad, $2^{\mathrm{a}}$ Ed. Madrid: Díaz de Santos, 2012, pp.23-84.

8. Rubens Sette Ramalho, Tratamiento de aguas residuales, $3^{\text {a }}$ Ed. Barcelona: Reverté, 2013, pp.582-587.

9. Metcalf \& Eddy, Wastewater Engineering: Treatment and Reuse, $4^{\mathrm{a}}$ Ed. New York: McGraw-Hill, 2003, pp. 659-861.

10. Rigola M, Tratamiento de aguas industriales: Aguas de proceso y aguas residuales, $2^{\mathrm{a}} \mathrm{Ed}$. Barcelona: Marcombo, 1999, pp.27-37 